Research report

\title{
Obstacle circumvention and eye coordination during walking to least and most affected side in people with Parkinson's disease
}

\author{
Fabio Augusto Barbieri ${ }^{\mathrm{a}, *}$, Paula Favaro Polastri ${ }^{\mathrm{a}}$, Lilian Teresa Bucken Gobbi ${ }^{\mathrm{b}}$, Lucas Simieli ${ }^{\mathrm{a}}$, \\ Vinicius Ignácio Alota Pereira ${ }^{a}$, André Macari Baptista ${ }^{a}$, Gabriel Felipe Moretto ${ }^{a}$, \\ Carolina Menezes Fiorellii, ${ }^{\mathrm{a}, \mathrm{c}}$, Luis Felipe Itikawa Imaizumi ${ }^{\mathrm{a}}$, Sérgio Tosi Rodrigues ${ }^{\mathrm{a}}$ \\ a São Paulo State University (Unesp) - Campus Bauru, Scholl of Science, Human Movement Research Laboratory (MOVI-LAB) and Laboratory of Information, Vision and \\ Action (LIVIA), Department of Physical Education, Bauru, SP, Brazil \\ ${ }^{\mathrm{b}}$ São Paulo State University (Unesp), Campus Rio Claro, Posture and Gait Studies Laboratory (LEPLO), Department of Physical Education, Rio Claro, SP, Brazil \\ ${ }^{\mathrm{c}}$ Universidade Sagrado Coração, Bauru, SP, Brazil
}

\section{A R T I C L E I N F O}

\section{Keywords:}

Gait

Vision

Basal ganglia

Asymmetry

Obstacle circumvention

Dopaminergic medication

\begin{abstract}
A B S T R A C T
Background: The mechanisms that contribute to gait asymmetry in people with Parkinson's disease (PD) are unclear, mainly during gait with greater environmental demand, such as when an obstacle is circumvented while walking.

Objective: The aim of this study was to investigate the effects of obstacle circumvention of the least and most affected side on motor and gaze behavior in people with PD under/without the effects of dopaminergic medication.

Methods: Fifteen people with PD and 15 matched-control individuals were instructed to walk along a pathway, at a self-selected velocity, and to circumvent an obstacle, avoiding contact with it. Each participant performed five trials for each side. Kinematic parameters, mediolateral and horizontal body clearance to the obstacle, strategy to circumvent the obstacle, and gaze behavior were calculated. Parameters were grouped according to the side that the obstacle was circumvented and compared by three-way ANOVAs.

Results: Both people with PD and the control group presented asymmetry to circumvent an obstacle during walking, however this was exacerbated in people with PD. Individuals with PD presented safe strategies (largest mediolateral and horizontal body clearance to the obstacle, "lead-out" strategy, and higher number and time of fixations on the obstacle) during obstacle circumvention for the least affected side compared to the most affected side. In addition, positive effects of dopaminergic medication on body clearance, spatial-temporal parameters, and gaze behavior were evidenced only when the obstacle was circumvented to the least affected side.

Conclusions: The obstacle circumvention to the most affected side is risky for people with PD.
\end{abstract}

\section{Introduction}

Obstacle circumvention is a more complex task than unobstructed walking. The former task requires that the individual detects the obstacle's position and edges, performs precise motor actions, and adjusts their movement around it, allowing adequate personal space (body clearance) at the point of moving past the obstacle to ensure safe navigation [1]. During obstacle circumvention, both people with Parkinson's disease (PD) and neurologically healthy individuals decrease step length and step velocity compared to unobstructed walking [2]. In addition, people with PD increase gait variability and duration of gaze fixations on the obstacle and ground when walking with obstacle circumvention, and reduce body clearance without effects from dopaminergic medication [2].

The planning and adjustments to circumvent an obstacle are according to the side of obstacle circumvention [3]. Previous studies have indicated that younger adults when performing circumvention of an obstacle during walking on the non-dominant side increase their personal space [3]. Preservation of body clearance to the obstacle is used as a control criterion by the locomotor system to plan motor adaptations, which is adjusted according to time required to acquire visual information and plan for upcoming hazards [3,4]. Circumvention of an obstacle to the non-dominant side seems to present slower information processing [5] that causes impairments (i.e. asymmetry) in the

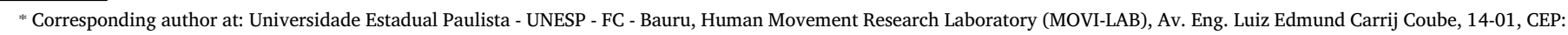
17033-360 Bauru, SP, Brazil.

E-mail address: barbieri@fc.unesp.br (F.A. Barbieri). 
acquisition and use of visual information [6] to make motor adjustments during the task. These impairments may be exacerbated in people with PD [7] who present symptoms manifestation more severely on one side [10-12] of the body from early stage of the disease [13-18]. Asymmetrical degeneration of dopaminergic neurons in the substantia nigra [19], enlarged lateral ventricle contralateral to the more symptomatic side [20], and cognitive disruption often consistent with the symptomatic hemisphere [21] may explain the higher effects in most affected side in people with PD. In addition, dopaminergic treatment has been established to improve gait motor patterns for both side of the body $[13,22]$, although levodopa has a greater effect on the most affected side $[23,24]$. However, no previous studies have investigated the effects of side to obstacle circumvention on body clearance to the obstacle, gait parameters and gaze behavior in people with PD.

The aim of this study was to investigate the effects on motor and visual behavior of obstacle circumvention during walking to the least and most affected side in people with PD, under and without the effects of dopaminergic medication. We analyzed the body clearance to the obstacle, circumvention strategy, spatial-temporal parameters, and gaze behavior during obstacle circumvention to both sides in people with PD and neurologically healthy individuals (control group). The hypotheses of this study were: i) people with PD would present safe strategies (increase body clearance, stride length and velocity and number of fixations on the obstacle) during obstacle circumvention to the side least affected by the disease compared to other side (most affected side) due to higher impairments presented in most affected side [13-18]; ii) dopaminergic medication would have a positive effect on body clearance, spatial-temporal adjustments and gaze behaviors (increase these parameters) for both sides during obstacle circumvention in people with $\mathrm{PD}$, as indicated previously in a study with obstacle avoidance [22].

\section{Materials and methods}

\subsection{Participants}

Fifteen people with idiopathic PD and 15 matched-neurologically healthy individuals (control group) were selected to participate in the study. The participants with PD were referred to the current study by local neurologists. The diagnosis of the disease was performed by an expert neurologist according to the UK Brain Bank Criteria $[27,28]$. The groups were matched by age, gender, body weight, and height (Table 1).

The following exclusion criteria were established: disease stage above 3 on the Hoehn \& Yahr scale [29,30], signs of dementia, a history of orthopedic or vision problems that would make it impossible to perform the experimental protocol. In addition, the inclusion criterion was the people with PD had to be taking PD medication. The study was approved by the local Ethics Committee (CAAE: 45435615.7.1001.5398). All participants gave their signed and written consent to all experimental procedures.

\subsection{Experimental protocol}

The individuals with PD performed the tasks in the OFF-medication state (after a minimum of $12 \mathrm{~h}$ withdrawal from PD medication), and then again $1 \mathrm{~h}$ after the participants had taken their dopaminergic medication (ON-medication state); if the individuals were taking dopaminergic agonist medication, they were evaluated after a minimum of $24 \mathrm{~h}$ withdrawal from medication. The control group performed the protocol only once.

\subsection{Clinical evaluation}

Participants with PD were evaluated by an expert researcher through anamneses (historical clinical, cognitive, and medication), the motor portion of the Unified Parkinson's Disease Rating Scale - UPDRS [31], and the H\&Y (stage of disease). In addition, cognitive aspects were analyzed using the Mini Mental State Exam [32,33] in all participants.

In addition, for people with PD, motor UPDRS items $20,21,22,23$, 25 , and 26 were used to assess appendicular asymmetry [14]. The most severely affected limb was determined by finding the difference between the scores for the right and left limbs in the aforementioned UPDRS items. Then, the values of this item-analysis were summed. When this calculation resulted in a positive value, the right limb was the most severely affected limb, but when negative values were obtained, this indicated that the left limb was more severely affected. For the control group, footedness was assessed by asking the participant to kick a ball at a target [21,23]. The limb used to kick the ball was considered as the dominant limb.

\subsection{Obstacle circumvention during gait}

The participants were instructed to walk along a pathway (approximately $8.5 \mathrm{~m}$ long by $3.5 \mathrm{~m}$ wide), at a self-selected velocity, and to circumvent an obstacle, avoiding contact with it. In addition, participants were instructed to return to the starting line. The obstacle was cylindrical $(0.35 \mathrm{~m}$ diameter) and $1.30 \mathrm{~m}$ high [2]. The obstacle was positioned in the middle of the pathway, allowing a similar space on both sides $(\sim 1.60 \mathrm{~m})$ and $4 \mathrm{~m}$ from the starting point. In all trials, the participant was positioned lined up with the obstacle.

Each participant performed 5 circumventions for each side (10 trials in total). The participants were not instructed as to which side they needed to circumvent the obstacle. They chose the side until they had performed 5 trials for one side (e.g., right). Then, the researcher obstructed this side, necessitating that the participants circumvent the obstacle on the other side (e.g., left).

\subsection{Data analysis}

The kinematic parameters were recorded by an 8 cameras Vicon Motion System (Bonita System Cameras) with a sample rate of 100 samples/s. Passive reflective markers were placed on the participants' skin at predefined landmarks according to the Plug-in-Gait Full Body model (Vicon) (left and right front and back head, 7th cervical vertebrae, 10th thoracic vertebrae, clavicle, sternum, middle of the right scapula, left and right shoulder, left and right upper arm, left and right elbow, left and right forearm, left and right wrist bar thumb and pinkie side, left and right fingers, left and right anterior and posterior superior iliac spine, mid-way between the posterior superior iliac spines, lateral epicondyle of the left and right knee, left and right lower lateral $1 / 3$ surface of the thigh, left and right lateral malleolus, left and right lower lateral $1 / 3$ of the shank, left and right second metatarsal head and left and right calcaneous) and four markers were placed on the obstacle. Data were filtered using a 5th order low-pass digital Butterworth filter (zero-lag) with a cutoff frequency of $6 \mathrm{~Hz}$.

The data were recorded in two phases of obstacle circumvention: the approach phase - final stride before circumvention of the obstacle; and circumvention phase - stride during the obstacle circumvention (Fig. 1). Nexus software (Vicon) calculated the tridimensional center of mass (CoM) coordinates based on the tridimensional coordinates of the 39 markers, which defined a 15-segment model [34]. Following CoM coordinates, we calculated the mediolateral body clearance (largest mediolateral distance of the CoM to the obstacle during obstacle circumvention) [35,36] (solid arrow in Fig. 2C) and the horizontal body clearance (distance at which participants started to circumvent the obstacle. To calculated this parameter, first it was drawn an imaginary line between the CoM position where participant began the trial and the marker positioned centrally in the top of the obstacle. So, the begin of deviation was defined as five standard deviations of this line) (dashed arrow in Fig. 2C). In addition, the following spatial-temporal parameters of gait for each phase were calculated: stride length, stride 
Table 1

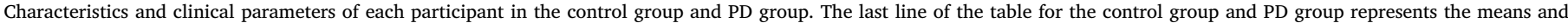
standard deviations of each group in each parameter. FP - foot preference.

\begin{tabular}{|c|c|c|c|c|c|c|c|c|c|c|c|}
\hline \multicolumn{12}{|l|}{ PD Group } \\
\hline & Sex & Age (years) & Body mass (kg) & Height (m) & $\mathrm{H} \& \mathrm{Y}$ OFF & H\&Y ON & MMSE OFF (pts) & MMSE ON (pts) & UPDRS III OFF (pts) & UPDRS III ON (pts) & FP \\
\hline 1 & M & 61 & 82.20 & 1.70 & 2 & 2 & 30 & 30 & 26 & 25 & $\mathrm{R}$ \\
\hline 2 & $\mathrm{~F}$ & 64 & 70.00 & 1.60 & 2 & 2 & 28 & 29 & 22 & 17 & $\mathrm{R}$ \\
\hline 3 & $\mathrm{~F}$ & 54 & 86.00 & 1.52 & 2 & 2 & 30 & 29 & 25 & 23 & $\mathrm{R}$ \\
\hline 4 & M & 61 & 50.30 & 1.63 & 2.5 & 2.5 & 29 & 30 & 47 & 39 & $\mathrm{R}$ \\
\hline 5 & $\mathrm{~F}$ & 74 & 48.00 & 1.41 & 3 & 3 & 20 & 21 & 60 & 48 & $\mathrm{R}$ \\
\hline 6 & $\mathrm{~F}$ & 74 & 64.80 & 1.61 & 1.5 & 1 & 26 & 26 & 26 & 16 & $\mathrm{R}$ \\
\hline 7 & $\mathrm{~F}$ & 64 & 85.70 & 1.55 & 1.5 & 1.5 & 30 & 28 & 26 & 24 & $\mathrm{R}$ \\
\hline 8 & $\mathrm{~F}$ & 52 & 70.70 & 1.49 & 2 & 2 & 27 & 30 & 41 & 32 & $\mathrm{R}$ \\
\hline 9 & M & 80 & 79.30 & 1.70 & 2 & 2 & 28 & 27 & 35 & 24 & $\mathrm{R}$ \\
\hline 10 & $\mathrm{~F}$ & 63 & 47.30 & 1.58 & 1 & 1 & 27 & 27 & 16 & 15 & $\mathrm{R}$ \\
\hline 11 & M & 75 & 61.50 & 1.67 & 2.5 & 2.5 & 25 & 25 & 39 & 33 & $\mathrm{R}$ \\
\hline 12 & $\mathrm{~F}$ & 84 & 67.00 & 1.60 & 3 & 3 & 28 & 28 & 25 & 24 & $\mathrm{R}$ \\
\hline 13 & M & 58 & 98.00 & 1.69 & 2 & 1.5 & 30 & 30 & 23 & 19 & $\mathrm{R}$ \\
\hline 14 & $\mathrm{~F}$ & 53 & 80.00 & 1.60 & 2 & 1 & 26 & 30 & 18 & 11 & $\mathrm{R}$ \\
\hline 15 & $\mathrm{~F}$ & 63 & 51.10 & 1.60 & 2.5 & 2.5 & 29 & 30 & 32 & 21 & $\mathrm{R}$ \\
\hline \multirow[t]{2}{*}{ Mean \pm SD } & $5 \mathrm{M} / 10 \mathrm{~F}$ & 65.33 & 69.46 & 1.59 & 2.10 & 1.97 & 27.53 & 28.00 & 30.73 & 24.73 & \\
\hline & & 9.90 & 15.75 & 0.08 & 0.54 & 0.67 & 2.64 & 2.54 & 11.84 & 9.77 & \\
\hline \multicolumn{12}{|c|}{ Control Group } \\
\hline & \multicolumn{3}{|c|}{$\operatorname{Sex}(M / F)$} & \multicolumn{2}{|l|}{ Age (years) } & \multicolumn{2}{|c|}{ Body mass (kg) } & Height (m) & \multicolumn{2}{|c|}{ MMSE (pts) } & FP \\
\hline 1 & \multicolumn{3}{|c|}{$\mathrm{F}$} & \multicolumn{2}{|l|}{74} & \multicolumn{2}{|l|}{63.50} & 1.53 & \multicolumn{2}{|l|}{29} & $\mathrm{R}$ \\
\hline 2 & \multicolumn{3}{|c|}{$\mathrm{F}$} & 56 & & \multicolumn{2}{|l|}{57.50} & 1.57 & \multicolumn{2}{|l|}{26} & $\mathrm{R}$ \\
\hline 3 & \multicolumn{3}{|c|}{ M } & 67 & & \multicolumn{2}{|l|}{76.50} & 1.67 & \multicolumn{2}{|l|}{29} & $\mathrm{R}$ \\
\hline 4 & \multicolumn{3}{|c|}{ M } & 74 & & \multicolumn{2}{|l|}{74.00} & 1.68 & 27 & & $\mathrm{R}$ \\
\hline 5 & & $\mathrm{~F}$ & & 65 & & 62.90 & & 1.65 & 29 & & $\mathrm{R}$ \\
\hline 6 & & M & & 67 & & 64.70 & & 1.69 & 27 & & $\mathrm{R}$ \\
\hline 7 & & $\mathrm{~F}$ & & 62 & & 80.30 & & 1.59 & 27 & & $\mathrm{R}$ \\
\hline 8 & & $\mathrm{~F}$ & & 71 & & 67.60 & & 1.57 & 27 & & $\mathrm{R}$ \\
\hline 9 & & $\mathrm{~F}$ & & 65 & & 76.50 & & 1.55 & 27 & & $\mathrm{R}$ \\
\hline 10 & & $\mathrm{~F}$ & & 65 & & 75.40 & & 1.54 & 24 & & $\mathrm{R}$ \\
\hline 11 & & M & & 77 & & 62.30 & & 1.62 & 28 & & $\mathrm{R}$ \\
\hline 12 & & $\mathrm{~F}$ & & 77 & & 77.80 & & 1.57 & 27 & & $\mathrm{R}$ \\
\hline 13 & & M & & 69 & & 88.00 & & 1.75 & 28 & & $\mathrm{~L}$ \\
\hline 14 & & $\mathrm{~F}$ & & 73 & & 51.00 & & 1.52 & 29 & & $\mathrm{R}$ \\
\hline 15 & & $\mathrm{~F}$ & & 66 & & 59.00 & & 1.56 & 25 & & $\mathrm{R}$ \\
\hline Mean \pm SD & & $5 \mathrm{M} / 10 \mathrm{~F}$ & & 68.53 & & 69.13 & & 1.60 & 27.27 & & \\
\hline & & & & 5.84 & & 10.12 & & 0.07 & 1.49 & & \\
\hline
\end{tabular}

width, stride duration, stride speed, and double support time (percentage of stride duration). The strategy chosen by the participants to circumvent the obstacle, "lead-out" (lead limb away from the obstacle during the crossing step) or "lead-in" (lead limb close to the obstacle during the crossing step) strategies, was also determined [35].

Gaze behavior was recorded by a mobile eye tracker (Mobile Eye- 5 glasses, ASL, Bedford, MA, USA). The frequency of data acquisition was $60 \mathrm{~Hz}$. The eye tracker system was calibrated using the nine-point calibration method. Participants fixated their gaze on nine points displayed in a $3 \times 3$ grid. Calibration was also checked periodically between trials. Gaze fixation was considered when the two times point of gaze standard deviation (95\% confidence interval) was less than one
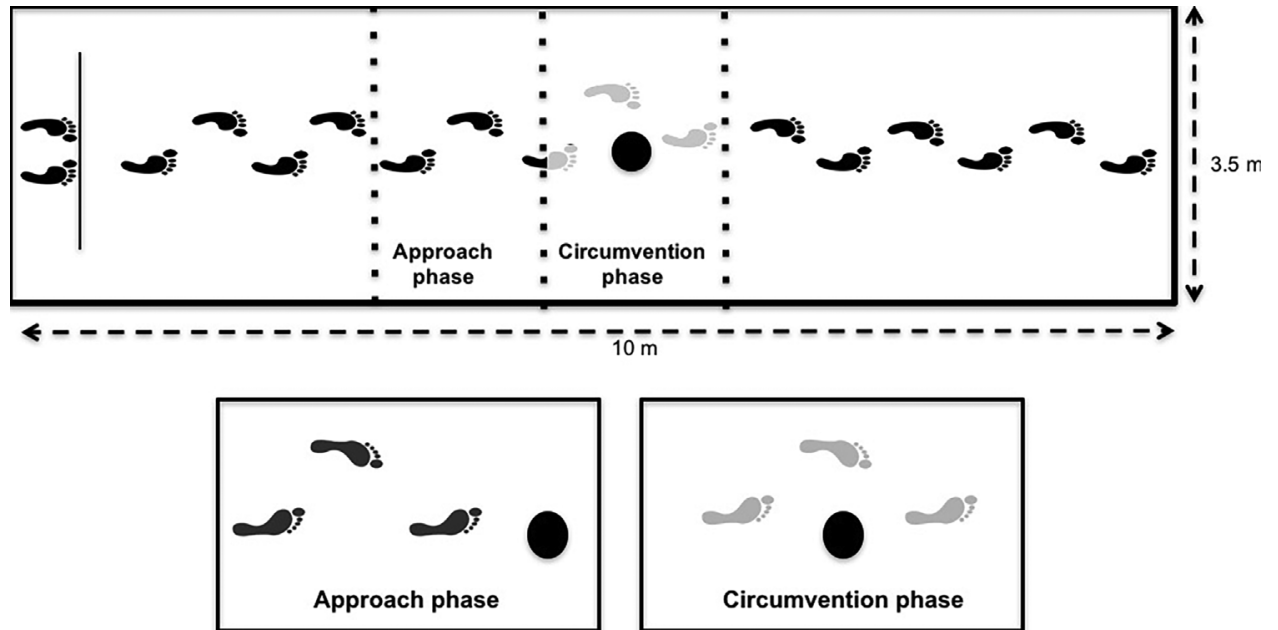

Fig. 1. Bird's eye view of the task when an individual performed obstacle circumvention to the left. The approach and circumvention phases are presented in the figure. 


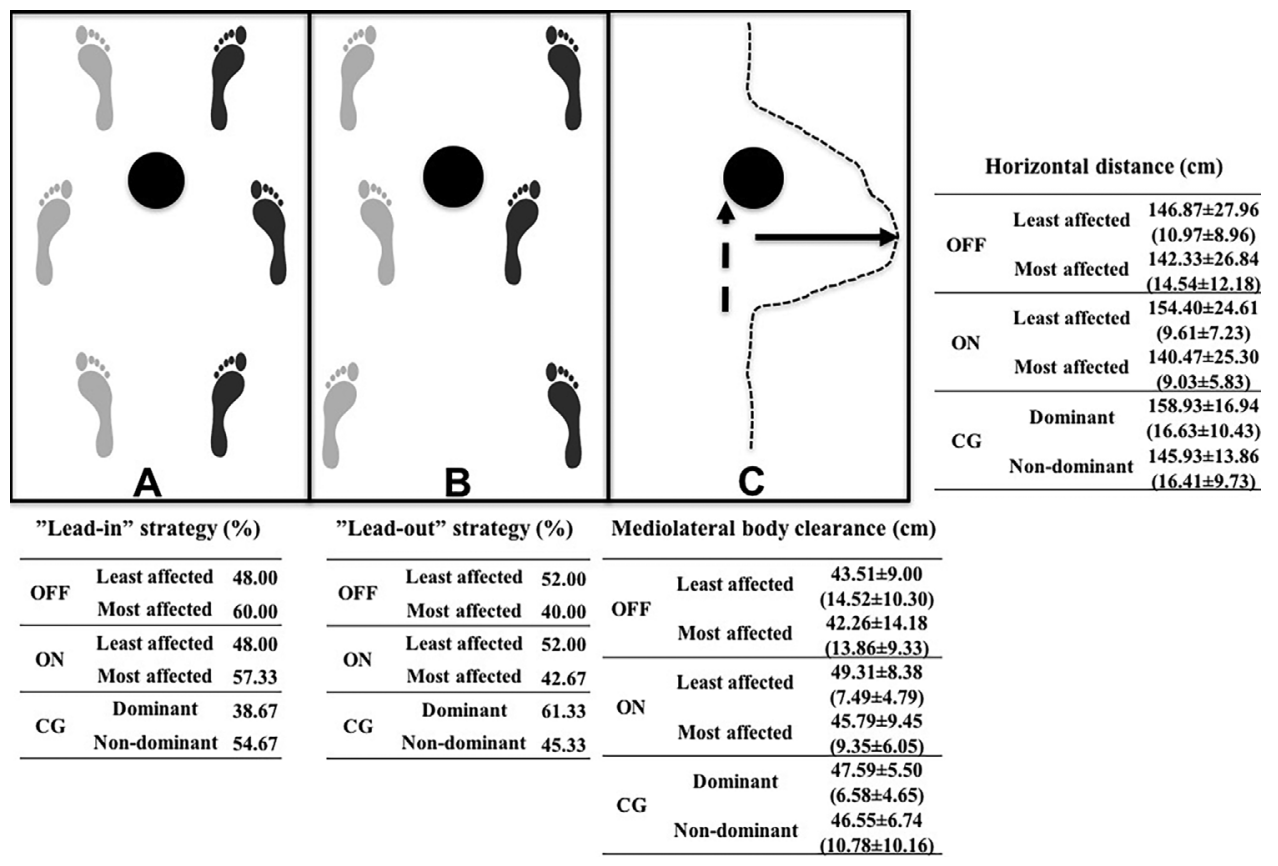

Fig. 2. A: Percentage of "lead-in" strategy according to side the obstacle was circumvented in people with PD under (ON) and without (OFF) the effects of dopaminergic medication. B: Percentage of "lead-out" strategy according to side the obstacle was circumvented in people with PD under and without the effects of dopaminergic medication. C: Means and standard deviations of mediolateral body clearance (solid arrow) and horizontal body clearance to the obstacle (dashed arrow) according to side the obstacle was circumvented in people with PD under and without the effects of dopaminergic medication. The values of variability (means and standard deviations) for each parameter are presented in brackets. degree of visual angle (horizontal and vertical) over $99 \mathrm{~ms}$. We analyzed the following parameters: number of fixations (total number of fixations during the trial), mean duration of the fixations, and time of fixations (percentage of the travel time) [8]. In addition, gaze fixations were classified into four different areas of interest: ground (any location on the ground before, after or to the side of the obstacle), obstacle (any location on the obstacle), wall (any area on the wall at the end of the walkway), and random (any areas not included in the other three areas). Finally, we determined the percentage of trials in which there were no fixations on the obstacle (no area of interest to the obstacle).

The parameters were grouped considering the side that the obstacle was circumvented during the task: people with PD - most and least affected side (defined by UPDRS items); control group - dominant and non-dominant side. In addition, the variability of each parameter was calculated. First, the average and standard deviation of each parameter was calculated according to conditions for each participant. Then, the variability was calculated from the coefficient of variation [37].

\subsection{Statistical analysis}

The level of significance was set at $5 \%$ for all analyzes. For clinical parameters, paired sample student $t$-tests were employed to compare dopaminergic medication effects (OFF-medication state - without dopaminergic medication effects $\times$ ON-medication state - under effects of dopaminergic medication). Independent sample student $t$-tests were employed to compare cognitive status of the control group and people with PD under the effects of dopaminergic medication. The spatialtemporal parameters, mediolateral and horizontal body clearance to the obstacle, and gaze behavior, as well as the variability of these parameters, were compared by two-way ANOVAs (group: PD patients under effects of dopaminergic medication and control group $\mathrm{X}$ side: least affected/dominant side and most affected/non-dominant side), separately for the approach phase and the circumvention phase, with side as repeated measure. For areas of interest, the data were analyzed by three-way ANOVAs (group X side X area of interest: ground, wall, obstacle, and random), with side and area of interest as repeated measures. A separate analysis for people with PD was conducted with dopaminergic status in a within-subject design with $\mathrm{ON}$ and $\mathrm{OFF}$ medication state as the repeated-measure (dopaminergic status comparison). In addition, Tukey post hoc tests were carried out to identify the significant differences when a significant main effect was found.

\section{Results}

\subsection{Clinical parameters}

The characteristics of both the PD and control groups and clinical features of the PD group under and without the effects of dopaminergic medication are presented in Table 1 . The groups presented no significant differences in cognitive aspects $\left(\mathrm{t}_{14}=1.36, \mathrm{p}=0.34\right)$. In addition, there were dopaminergic medication effects in UPDRS-motor, in which people with PD presented the lowest score (improvement in UPDRS-motor) under the effects of dopaminergic medication $\left(t_{14}=5.75, p \ll 0.001\right)$. There were no effects of dopaminergic medication on $\mathrm{H} \& \mathrm{Y} \quad\left(\mathrm{t}_{14}=1.74, \mathrm{p}=0.11\right)$ or cognitive aspects $\left(\mathrm{t}_{14}=-1.20, \mathrm{p}=0.25\right)$.

\subsection{Obstacle circumvention strategy, body clearance, and spatial-temporal parameters}

There was not a preferred side (most/non-dominant or least/ dominant side) to circumvent the obstacle for both groups. For control group, five participants, two participants and eight participants performed the first five obstacle circumvention to, respectively, dominant side, non-dominant side and randomly (i.e. two for one side and for the another side). For people with PD, two participants performed the first five obstacle circumvention to least affected side (five participants in OFF-medication state), one participant performed the first five obstacle circumvention to most affected side (two participants in OFF-medication state) and twelve participants performed the first five trials randomly (eight participants in OFF-medication state).

Regarding obstacle circumvention strategies, people with PD used the "lead-in" strategy more when they performed obstacle circumvention to the most affected side while the control group used the "lead-in" strategy and "lead-out" strategy more when they performed obstacle circumvention to the dominant side and non-dominant side, respectively (Fig. 2).

Both groups increased the horizontal body clearance to the obstacle (beginning the obstacle circumvention) when they performed obstacle circumvention to the least affected/dominant side $\left(\mathrm{F}_{1,28}=14.89\right.$, 


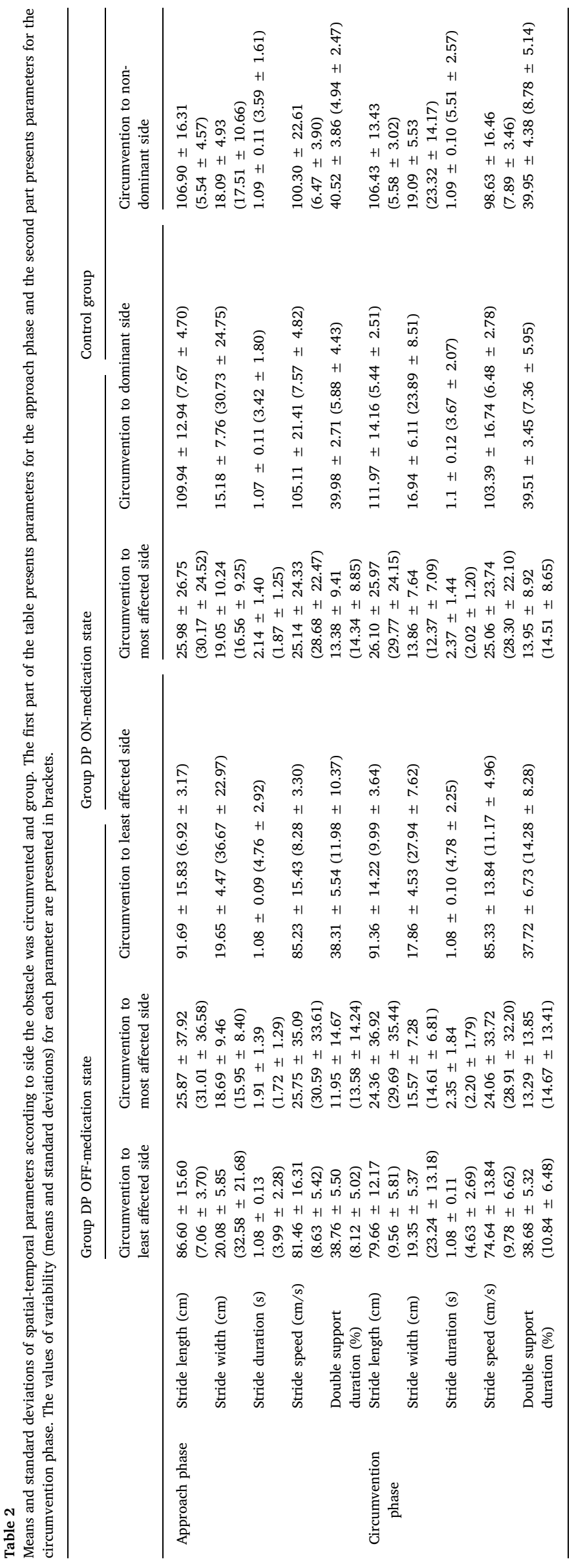

$\mathrm{p} \ll 0.001$ ) (Fig. 2). In addition, people with PD presented lower variability in horizontal body clearance to the obstacle than the control group for obstacle circumvention to the least affected/dominant side $\left(F_{1,28}=7.61, p \ll 0.01\right)$. With respect to mediolateral body clearance to the obstacle, people with PD increased the clearance to the obstacle ( $\mathrm{p} \ll 0.008$ ) when they performed obstacle circumvention to the least affected side compared to obstacle circumvention to the most affected side (group*side interaction $-\mathrm{F}_{1,28}=4.95, \mathrm{p} \ll 0.004$ ). The control group did not present significant differences between sides for mediolateral body clearance, however they increased variability of mediolateral body clearance $(\mathrm{p} \ll 0.04)$ for non-dominant obstacle circumvention compared to dominant obstacle circumvention (group*side interaction $-\mathrm{F}_{1,28}=3.42$, $\mathrm{p} \ll 0.02$ ).

People with PD presented slower stride velocity (approach phase $\mathrm{F}_{1,28}=6.08, \quad \mathrm{p} \ll 0.02 ; \quad$ circumvention phase $-\mathrm{F}_{1,28}=8.07$, $\mathrm{p} \ll 0.008$ ), shorter stride length (approach phase $-\mathrm{F}_{1,28}=10.01$, $\mathrm{p} \ll 0.004$; circumvention phase $-\mathrm{F}_{1,28}=12.70, \mathrm{p} \ll 0.001$ ), and higher variability of double support time (approach phase $\mathrm{F}_{1,28}=6.71, \mathrm{p} \ll 0.01$; circumvention phase $-\mathrm{F}_{1,28}=5.39, \mathrm{p} \ll 0.02$ ) in both the approach and circumvention phases than the control group (Table 2). Specifically for the approach phase, people with PD increased stride width $\left(\mathrm{F}_{1,28}=8.08, \mathrm{p} \ll 0.008\right)$ and variability of this parameter $\left(\mathrm{F}_{1,28}=5.47, \mathrm{p} \ll 0.02\right)$ compared to the control group. In addition, when the individuals performed the obstacle circumvention to the most affected/non-dominant side, people with PD increased variability of stride width ( $\mathrm{p} \ll 0.03$ ) compared to the control group in the approach phase (group*side interaction $-\mathrm{F}_{1,28}=10.33$, $\mathrm{p} \ll 0.003$ ). For the circumvention phase, people with PD increased variability of stride length $\left(\mathrm{F}_{1,28}=7.63, \mathrm{p} \ll 0.01\right)$ and velocity $\left(\mathrm{F}_{1,28}=3.88, \mathrm{p} \ll 0.05\right)$ compared to the control group. In addition, when individuals performed obstacle circumvention to the least affected/dominant side, people with $P D$ increased variability of stride length ( $\mathrm{p} \ll 0.001)$, velocity ( $\mathrm{p} \ll 0.003$ ), and double support time ( $\mathrm{p} \ll 0.01$ ) compared to the control group (group*side interaction: stride length $-\mathrm{F}_{1,28}=3.53$, $\mathrm{p} \ll 0.05$; stride velocity $-\mathrm{F}_{1,28}=7.16$, $\mathrm{p} \ll 0.01$; double support time $\left.-\mathrm{F}_{1,28}=3.59, \mathrm{p} \ll 0.05\right)$. People with $\mathrm{PD}$ also increased variability of stride length $(\mathrm{p} \ll 0.01)$ and velocity $(\mathrm{p} \ll 0.01)$ when they performed obstacle circumvention to the least affected side compared to the most affected side, while the control group reduced variability of stride duration $(\mathrm{p} \ll 0.05)$ when they circumvented the obstacle to the dominant side compared to non-dominant side (group*side interaction: variability of stride duration $-\mathrm{F}_{1,28}=3.67, \mathrm{p} \ll 0.05$ ). Finally, the control group was faster (increased stride velocity) in both phases (approach phase $-\mathrm{p} \ll 0.01$; circumvention phase $-\mathrm{p} \ll 0.05$ ) when they performed obstacle circumvention to the dominant side compared to the non-dominant side.

\subsection{Gaze behavior and area of interest}

People with PD presented higher variability of time of fixations $\left(F_{1,28}=4.22, p \ll 0.04\right)$ compared to the control group (Table 3$)$. In addition, when individuals performed obstacle circumvention to the least affected/dominant side, they increased variability of mean duration of fixations $\left(\mathrm{F}_{1,28}=5.35, \mathrm{p} \ll 0.02\right)$. Post hoc analysis of group*side interaction (mean duration of fixation $-\mathrm{F}_{1,28}=4.82$, $\mathrm{p} \ll 0.03$; variability of time of fixations $-\mathrm{F}_{1,28}=10.98, \mathrm{p} \ll 0.003$ ) indicated that when the obstacle circumvention was performed to the most affected/non-dominant side, people with PD increased the mean duration of fixation $(\mathrm{p} \ll 0.04)$ and variability of time of fixations $(\mathrm{p} \ll 0.03)$ compared to the control group. In addition, the control group presented higher time of fixations $(\mathrm{p} \ll 0.04)$ when they circumvented the obstacle to the dominant side (group*side interaction $-\mathrm{F}_{1,28}=4.82$, $\mathrm{p} \ll 0.03)$.

In general, for area of interest, individuals presented a higher number of fixations $\left(\mathrm{F}_{1,28}=36.52, \mathrm{p} \ll 0.001\right)$ and time of fixations $\left(F_{1,28}=43.56, p \ll 0.001\right)$ on the ground compared to other areas of 
interest (obstacle, wall, and random areas) (Table 3). The number of fixations $(\mathrm{p} \ll 0.001)$ and time of fixations $(\mathrm{p} \ll 0.001)$ on the obstacle was higher than on the wall and random. In addition, the mean duration of fixations $\left(F_{1,28}=9.13, p \ll 0.001\right)$ on the obstacle and ground was higher than random. When individuals performed obstacle circumvention to the most affected/non-dominant side, they decreased the number of fixations $(\mathrm{p} \ll 0.002)$ and time of fixations $(\mathrm{p} \ll 0.004)$ on the obstacle, and increased the number of fixations $(\mathrm{p} \ll 0.002)$, time of fixations ( $\mathrm{p} \ll 0.002)$, and mean duration of fixations ( $\mathrm{p} \ll 0.01)$ on the ground compared to obstacle circumvention to the least affected/ dominant side (side*area interaction - number of fixations: $\mathrm{F}_{1,28}=13.56, \mathrm{p} \ll 0.001$; mean duration of fixations: $\mathrm{F}_{1,28}=6.31$, $\mathrm{p} \ll 0.001$; time of fixations: $\mathrm{F}_{1,28}=13.24, \mathrm{p} \ll 0.001$ ). Furthermore, when obstacle circumvention was performed to the most affected/nondominant side, they performed a higher number of fixations ( $\mathrm{p} \ll 0.001)$ and time of fixations $(\mathrm{p} \ll 0.001)$ on the ground than the other three areas of interest, while when obstacle circumvention was performed to the least affected/dominant side, they performed the same number of fixations $(\mathrm{p} \ll 0.001)$ and time of fixations $(\mathrm{p} \ll 0.001)$ on the obstacle and ground, but both higher than on the wall and random.

Specifically, people with PD presented a higher number of fixations $(\mathrm{p} \ll 0.001)$ and time of fixations $(\mathrm{p} \ll 0.002)$ on the obstacle than the control group (group*area interaction - number of fixations: $\mathrm{F}_{1,28}=5.01, \mathrm{p} \ll 0.02$; time of fixations: $\left.\mathrm{F}_{1,28}=7.78, \mathrm{p} \ll 0.003\right)$. In addition, people with PD increased the number of fixations ( $\mathrm{p} \ll 0.001)$, mean duration of fixations ( $\mathrm{p} \ll 0.04$ ), and time of fixations $(\mathrm{p} \ll 0.001)$ on the obstacle when performing obstacle circumvention to the least affected limb compared to the control group when circumventing the obstacle to the dominant side (group*side*area interaction - number of fixations: $\mathrm{F}_{1,28}=17.20, \mathrm{p} \ll 0.001$; mean duration of fixations: $\mathrm{F}_{1,28}=4.29, \mathrm{p} \ll 0.01$; time of fixations: $\left.\mathrm{F}_{1,28}=24.56, \mathrm{p} \ll 0.001\right)$. For the other side (most affected and non-dominant side), people with PD increased the mean duration of fixations $(\mathrm{p} \ll 0.002)$ on the ground compared to the control group. Furthermore, when people with PD circumvented the obstacle to the most affected side, they decreased the number of fixations $(\mathrm{p} \ll 0.001)$ and time of fixations $(\mathrm{p} \ll 0.001)$ on the obstacle and increased the number of fixations ( $\mathrm{p} \ll 0.001)$, mean duration of fixations $(\mathrm{p} \ll 0.001)$, and time of fixations $(\mathrm{p} \ll 0.001)$ on the ground; they did not fixate the obstacle in $62.66 \%$ of trials when circumventing the obstacle to the most affected side (for least affected side only $9.3 \%$ of trials). In contrast, the control group presented similarity in this variable for both sides of obstacle circumvention (dominant side $-62.66 \%$ of trials; non-dominant side $-48 \%$ of trials).

\subsection{ON-medication state vs OFF-medication state}

People with PD increased the use of a "lead-in" strategy when performing obstacle circumvention to the most affected side, independent of the effects (both under and without) of dopaminergic medication (Fig. 2). However, they used a similar number of "lead-in" and "lead-out" strategies when performing obstacle circumvention to the least affected side, independent of the effects of dopaminergic medication. In addition, the lack of dopaminergic medication increased the variability of mediolateral body clearance $\left(F_{1,28}=5.78, p \ll 0.02\right)$, especially when circumventing the obstacle to the least affected side (dopaminergic status*side interaction $-\mathrm{F}_{1,28}=5.74, \mathrm{p} \ll 0.02$ ) (Fig. 2). Furthermore, under the effects of dopaminergic medication, people with PD began $(\mathrm{p} \ll 0.04)$ the obstacle circumvention earlier (horizontal body clearance) than when they performed obstacle circumvention to the least affected side compared to the most affected side (medication*side interaction $-\mathrm{F}_{1,28}=3.84, \mathrm{p} \ll 0.05$ ).

There were no effects of dopaminergic medication, side or interaction between factors for spatial-temporal parameters of obstacle circumvention during the approach phase (Table 2). During the obstacle circumvention phase, the lack of dopaminergic medication decreased stride length $(\mathrm{p} \ll 0.02)$ and velocity $(\mathrm{p} \ll 0.04)$ when circumventing the obstacle to the least affected side - compared to the ON-dopaminergic medication state (medication*side interaction - stride length: $\mathrm{F}_{1,28}=3.97, \mathrm{p} \ll 0.05 ;$ stride velocity: $\left.\mathrm{F}_{1,28}=4.47, \mathrm{p} \ll 0.04\right)$. Furthermore, when the people with $\mathrm{PD}$, without the effects of dopaminergic medication, performed obstacle circumvention to the most affected side, they increased stride length $(\mathrm{p} \ll 0.006)$ and velocity ( $\mathrm{p} \ll 0.02)$ compared to the least affected side. However, dopaminergic medication increased the variability of stride length $(\mathrm{p} \ll 0.05)$ and velocity ( $\mathrm{p} \ll 0.03$ ) when people with PD circumvented the obstacle to the least affected side compared to the most affected side (medication*side interaction - stride length: $\mathrm{F}_{1,28}=4.77, \mathrm{p} \ll 0.03$; stride velocity: $F_{1,28}=5.20, p \ll 0.03$ ).

There were no effects of dopaminergic medication, side or interaction between factors for gaze behavior (Table 3). However, for areas of interest, the lack of dopaminergic medication decreased the number of fixations $(\mathrm{p} \ll 0.006)$ and time of fixations $(\mathrm{p} \ll 0.009)$ on the obstacle and increased the mean duration of fixations $(\mathrm{p} \ll 0.02)$ on the ground (medication*area interaction - number of fixations: $\mathrm{F}_{1,28}=4.62$, $\mathrm{p} \ll 0.03$; time of fixations: $\mathrm{F}_{1,28}=5.82, \mathrm{p} \ll 0.01$; mean duration of fixations: $F_{1,28}=5.79, p \ll 0.02$ ). In addition, the lack of dopaminergic medication increased the number of fixations $(\mathrm{p} \ll 0.002)$, mean duration of fixations $(\mathrm{p} \ll 0.003)$, and time of fixations $(\mathrm{p} \ll 0.001)$ on the ground, and reduced the number of fixations $(\mathrm{p} \ll 0.001)$ and time of fixations $(p \ll 0.001)$ on the obstacle when the obstacle was circumvented to the least affected side - compared to under the effects of dopaminergic medication (medication*side*area interaction - number of fixations: $\mathrm{F}_{1,28}=18.50, \mathrm{p} \ll 0.001$; mean duration of fixations: $\mathrm{F}_{1,28}=3.27, \mathrm{p} \ll 0.02$; time of fixations: $\mathrm{F}_{1,28}=24.26, \mathrm{p} \ll 0.001$ ). In addition, the mean duration of fixations on the obstacle decreased ( $\mathrm{p} \ll 0.04$ ) when they performed obstacle circumvention to the most affected side compared to the least affected side without the effects of dopaminergic medication. Dopaminergic medication increased the number of fixations $(\mathrm{p} \ll 0.001)$, mean duration of fixations ( $\mathrm{s} \ll 0.001$ ), and time of fixations $(\mathrm{p} \ll 0.001)$ on the ground and decreased the number of fixations $(\mathrm{p} \ll 0.001)$ and time of fixations ( $\mathrm{p} \ll 0.001$ ) on the obstacle when people with PD performed obstacle circumvention to the most affected limb compared to obstacle circumvention to the least affected limb. Finally, people with PD without the effects of dopaminergic medication did not fixate the obstacle in $41.33 \%$ and $48 \%$ of trials when circumventing the obstacle to the least and most affected sides, respectively. The percentage was greater for the least affected side and lower for the most affected side compared to under the effects of dopaminergic medication (see end of Section 3.3).

\section{Discussion}

The aim of this study was to investigate the effects on body clearance, spatial-temporal adjustments, and gaze behavior of obstacle circumvention to the least and most affected side in people with PD, under and without the effects of dopaminergic medication, and compare these effects with neurologically healthy individuals. People with PD and the control group presented similar strategies to circumvent an obstacle (both used "lead-in" and "lead-out" strategies, began obstacle circumvention at a similar horizontal body clearance to the obstacle, and had similar mediolateral body clearance from the obstacle), which corroborated with our previous study [2]. However, the groups performed different spatial-temporal and gaze adjustments to circumvent the obstacle. People with PD reduced gait velocity and stride length during the approach and circumvention phases, which may be due to hypometric movements and bradykinesia caused by PD [7,9]. This strategy suggests an increased time to acquire information on the environment [38], mainly the obstacle. People with PD were more gaze obstacle-dependent (they increased the number and time of fixations on the obstacle). Vitório and collaborators [39] demonstrated that environmental constraints, such as postural threat, increase the dependence of people with PD on dynamic visual information during 
locomotion. Sensory and perceptual deficits caused by PD [40-42] are the main explanation for this gaze behavior during obstacle circumvention. Basal ganglia damage may be associated with sensory and perceptual deficits $[43,44]$ as well as right hemisphere damage, which is responsible for visuospatial processing [45]. On the other hand, reduced gait velocity and stride length increases instability during walking [46-48]. To deal with this unstable gait, people with PD increased the basis of support (stride width) during the approach phase. In addition, the greater variability in stride width and double support time could be an attempt by the system to seek stability. Despite this, a large variability (besides stride width and double support time, people with PD increased variability of stride length and velocity during the approach phase), may suggest impairment in both mechanisms that regulate gait rhythm and the central pattern generator and those that regulate balance [49] and sequential and rhythmic movements caused by lesions in the posterior part of the putamen [42,50]. The greater variability in people with PD could be interpreted, from a pathophysiology aspect, as a deficit in the basal ganglia internal rhythmicity [51,52].

\subsection{Strategies of people with PD to deal with asymmetry when circumventing the obstacle}

Our most important finding was that people with PD presented asymmetry when circumventing the obstacle, which confirmed our first hypothesis. People with PD presented safe strategies (greater mediolateral and horizontal body clearance to the obstacle, "lead-out" strategy, and higher number and time of fixations on the obstacle) during obstacle circumvention to the least affected side compared to the most affected side, which seems to indicate asymmetric motor and gaze behavior to circumvent an obstacle. However, neurologically healthy individuals also presented asymmetric behavior, although lower than people with $\mathrm{PD}$, in the obstacle circumvention strategy, body clearance, spatial-temporal, and gaze strategies, which seems to indicate that asymmetric behavior during obstacle circumvention begins in neurologically healthy individuals and is exacerbated in people with PD. A previous study [3] related asymmetric behavior during obstacle circumvention in younger and older adults. The explanation for this asymmetric behavior is that visual-spatial information is processed faster when obstacle circumvention is performed to the dominant side due to the greater cortical representation and salience of this side [53].

People with PD performed a risky strategy when circumventing an obstacle to the most affected side. They began the obstacle circumvention closer to the obstacle (shorter horizontal body clearance), used a "lead-in" strategy and smaller mediolateral body clearance compared to when the obstacle was circumvented to the least affected side. In addition, they increased the number and time of fixations on the ground and decreased these parameters on the obstacle when circumventing the obstacle to the most affected side. These findings reinforce the risky strategy, which can increase the chances of making contact with the obstacle. Obstacle circumvention to the most affected side seemed to increase the need to guarantee the accuracy and precision of foot placement and the path to obstacle circumvention. To deal with this need is the reduced gait velocity during approach and circumvention phases during obstacle circumvention to the most affected side, which increased the time to plan the foot position and to place the foot precisely [48,54]. Due to the proprioceptive [43] and working memory [55] deficits usually observed in people with $\mathrm{PD}$, they are more dependent on the availability of visual information in an on-line mode to fine tune the accuracy of foot placement [8]. Therefore, asymmetric control of the basal ganglia seems to be evidenced due to the fact that the brain side that controls the most affected limb seems to present worse striatal uptake in both the caudate and putamen nuclei [56,57] and reduced distribution throughout the cortical-basal ganglia-thalamic circuitry $[58,59]$. In addition, the deficits in circumventing the obstacle to the most affected side were not only in the motor system, but also in the sensorial system. We can speculate that degeneration in the most affected brain side also occurred in perceptual brain areas. Previous studies have also indicated that veering is side affected to PD-dependent $[60,61]$. Perceptual/sensorial asymmetry is a characteristic in the earliest stages of the disease $[54,59,62,63]$, which seems to be manifested depending on the basal ganglia-cortical loops activated by the visual and motor demands of the task [62]. Finally, obstacle circumvention to the most affected side decreased the variability of stride length and velocity. This strategy seems to indicate a greater robustness of the motor system that can be explained by the difficulty of people with PD to perform adjustments during challenging tasks due to impairments in the basal ganglia, decreasing motor flexibility - action reprogramming [37], which seems to be a worse strategy if an adaptation is necessary during the task [22].

\subsection{No effect of dopaminergic medication during obstacle circumvention to the most affected side}

The second main important finding of our study was that dopaminergic medication presented no effects on motor or perceptual systems for obstacle circumvention to the most affected side. Therefore, our second hypothesis was only confirmed when people with PD performed obstacle circumvention to the least affected side, which presented positive effects from dopaminergic medication on mediolateral body clearance (reduced variability), spatial-temporal parameters (greater stride length during circumvention phase), and gaze behavior (higher number and longer fixations on the obstacle). However, our analysis did not reveal effects from dopaminergic medication when obstacle circumvention was performed to the most affected side. These results suggest that effects from dopaminergic medication were dependent on the side that people with PD performed obstacle circumvention. No symmetrical action of dopaminergic medication can explain our findings $[23,64]$. Previous studies have demonstrated that there are differences in cerebral activity between the most and least affected sides and that levodopa has a preferential effect on brain activations in taskrelevant brain areas [21]. The least affected side might have preferentially responded to levodopa because the nigrostriatal dopaminergic terminals of that side were less degenerated [64]. These findings corroborate with our previous study that found PD medication improved postural control asymmetry in people with PD in the early stage of disease, but not in the moderate stage, during challenging postural tasks [26]. Asymmetrical degeneration of dopaminergic neurons in the substantia nigra, such as contralateral lateral ventricle is enlarged to the most symptomatic side and hypoactivity in motor regions is higher in the most affected side, underlying symptom asymmetry in PD $[18,19]$. The advanced brain impairment due to PD presents a greater response with shorter duration resulting in waxing and waning between medication doses, and eventually, abrupt changes in response as if turned on and off by a switch $[65,66]$ which results in delayed onset to the most affected side compared with the least affected side [66]. In addition, no effects from dopaminergic medication during obstacle circumvention to the most affected side occurred in the perceptual system, which seems to indicate that dopaminergic circuits within the basal ganglia may not be responsible for perceptual deficits, corroborating with previous studies $[41,67,68]$. Therefore, obstacle circumvention to the most affected side is risky task, which could have a contact with the obstacle in people with PD without the effects of dopaminergic medication.

In conclusion, both people with PD and neurologically healthy individuals presented asymmetric behavior between sides when circumventing the obstacle, however, this asymmetry was exacerbated in the former. People with PD performed worse motor and perceptual strategies during obstacle circumvention to the most affected side compared to the least affected side. In addition, dopaminergic medication had no effects on motor or perceptual systems for obstacle circumvention to the most affected side. The effects from dopaminergic medication occurred only when obstacle circumvention was performed 


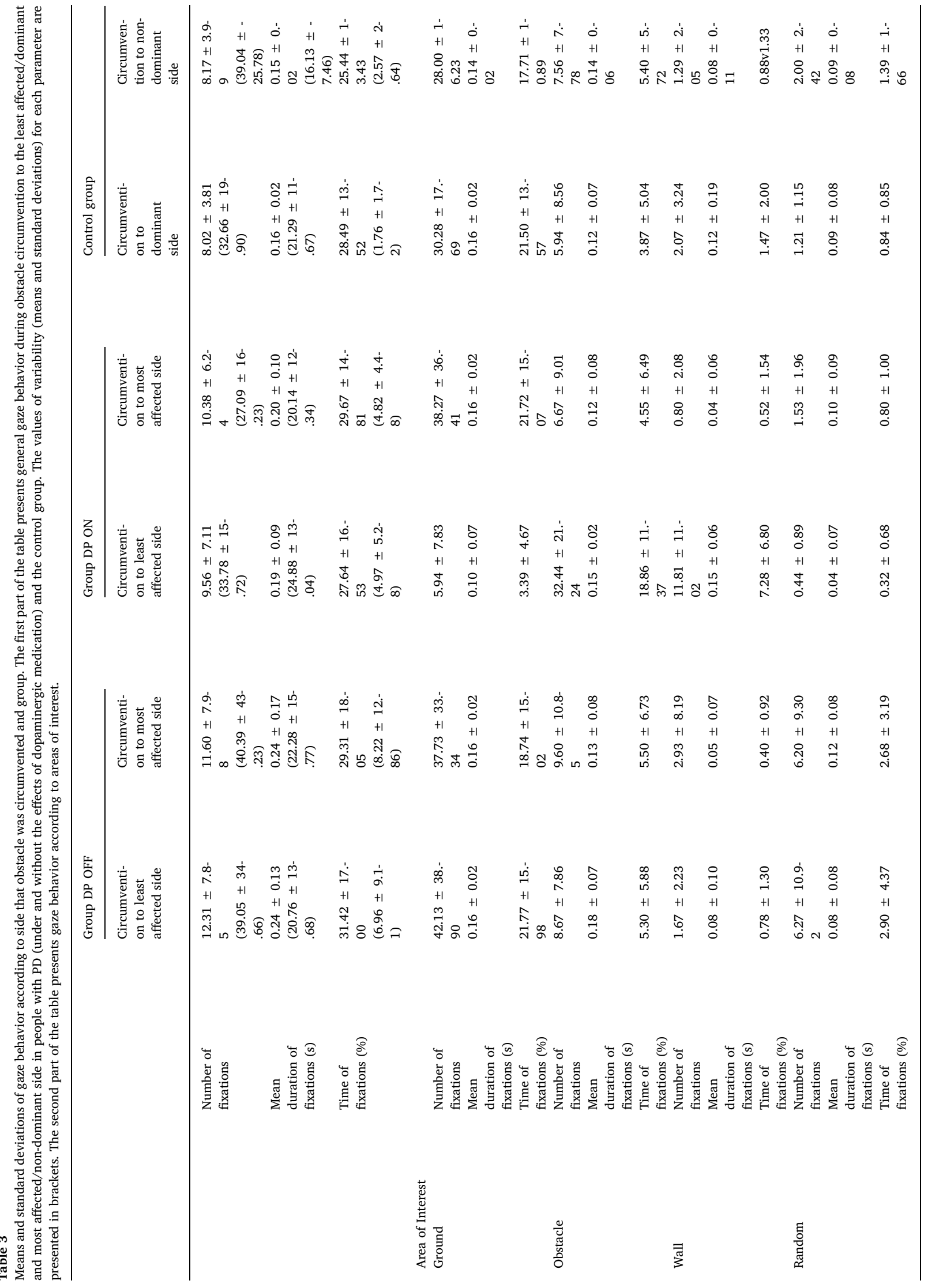


to the least affected side. Therefore, obstacle circumvention to the most affected side is risky for people with PD, mainly without the effects of dopaminergic medication. Future studies should investigate the relationship between side preference and side (most) affected by the disease to advance discussions about degeneration of the brain.

\section{Conflict of interests}

The authors have no conflict of interest to report.

\section{Acknowledgements}

This work was supported by the São Paulo Research Foundation (FAPESP) [grant numbers 2014/20549-0; 2015/15928-4; 2016/098050]; the Pró-Reitoria de Pesquisa da UNESP (PROPE) [grant numbers 76/ 2015;07/2016].

\section{References}

[1] G. Yogev, M. Plotnik, C. Peretz, N. Giladi, J.M. Hausdorff, Gait asymmetry in patients with Parkinson's disease and elderly fallers: when does the bilateral coordination of gait require attention? Exp. Brain Res. 177 (2007) 336-346.

[2] L. Simieli, R. Vitorio, S.T. Rodrigues, P.F.P. Zago, V.A.I. Pereira, A.M. Baptista, P.H.A. de Paula, T. Penedo, Q.J. Almeida, F.A. Barbieri, Gaze and motor behavior of people with PD during obstacle circumvention, Gait Posture 58 (2017) 504-509.

[3] M. Gérin-Lajoie, C.L. Richards, J. Fung, B.J. McFadyen, Characteristics of personal space during obstacle circumvention in physical and virtual environments, Gait Posture 27 (2008) 239-247.

[4] M. Gérin-Lajoie, C.L. Richards, B.J. McFadyen, The circumvention of obstacles during walking in different environmental contexts: a comparison between older and younger adults, Gait Posture 24 (2006) 364-369.

[5] S. Coren, C. Porac, Monocular asymmetries in visual latency as a function of sighting dominance, Am. J. Optom. Physiol. Opt. 59 (1982) 987-990.

[6] R.A. Armstrong, Visual symptoms in Parkinson's disease, Parkinson Dis. 2011 (2011) 908306.

[7] R. Vitório, F. Pieruccini-Faria, F. Stella, S. Gobbi, L.T.B. Gobbi, Effects of obstacle height on obstacle crossing in mild Parkinson's disease, Gait Posture 31 (2010) 143-146.

[8] R. Vitório, E. Lirani-Silva, F. Pieruccini-Faria, R. Moraes, L.T.B. Gobbi, Q.J. Almeida, Visual cues and gait improvement in Parkinson's disease: which piece of information is really important? Neuroscience 277 (2014) 273-280.

[9] F. Pieruccini-Faria, R. Vitório, Q.J. Almeida, C.R.A. Silveira, M.J.D. Caetano, F. Stella, S. Gobbi, L.T.B. Gobbi, Evaluating the acute contributions of dopaminergic replacement to gait with obstacles in Parkinson's disease, J. Mot. Behav. 45 (2013) 369-380.

[10] S.T. Moore, H.G. MacDougall, W.G. Ondo, Ambulatory monitoring of freezing of gait in Parkinson's disease, J. Neurosci. Meth. 167 (2008) 340-348.

[11] H. Sadeghi, P. Allard, F. Prince, H. Labelle, Symmetry and limb dominance in ablebodied gait: a review, Gait Posture 12 (2000) 34-45.

[12] J.C. Wall, G.I. Turnbull, Gait asymmetries in residual hemiplegia, Arch. Phys. Med. Rehabil. 67 (1986) 550-553.

[13] M. Plotnik, N. Giladi, Y. Balash, C. Peretz, J.M. Hausdorff, Is freezing of gait in Parkinson's disease related to asymmetric motor function? Ann. Neurol. 57 (2005) $656-663$.

[14] R.J. Uitti, Y. Baba, N.R. Whaley, Z.K. Wszolek, J.D. Putzke, Parkinson disease: handedness predicts asymmetry, Neurology 64 (2005) 1925-1930.

[15] S. Yust-Katz, D. Tesler, T.A. Treves, E. Melamed, R. Djaldetti, Handedness as a predictor of side of onset of Parkinson's disease, Parkinsonism Relat. Disord. 14 (2008) 633-635.

[16] K.C. Stewart, H.H. Fernandez, M.S. Okun, R.L. Rodriguez, C.E. Jacobson, C.J. Hass, Side onset influences motor impairments in Parkinson disease, Parkinsonism Relat. Disord. 15 (2009) 781-783.

[17] R. Baltadjieva, N. Giladi, L. Gruendlinger, C. Peretz, J.M. Hausdorff, Marked alterations in the gait timing and rhythmicity of patients with de novo Parkinson's disease, Eur. J. Neurosci. 24 (2006) 1815-1820.

[18] R. Djaldetti, I. Ziv, E. Melamed, The mystery of motor asymmetry in Parkinson's disease, Lancet Neurol. 5 (2006) 796-802.

[19] P.A. Kempster, W.R. Gibb, G.M. Stern, A.J. Lees, Asymmetry of substantia nigra neuronal loss in Parkinson's disease and its relevance to the mechanism of levodopa related motor fluctuations, J. Neurol. Neurosurg. Psychiatry 52 (1989) 72-76.

[20] M.M. Lewis, A.B. Smith, M. Styner, H. Gu, R. Poole, H. Zhu, Y. Li, X. Barbero, S. Gouttard, M.J. McKeown, R.B. Mailman, X. Huang, Asymmetrical lateral ventricular enlargement in Parkinson's disease, Eur. J. Neurol. 16 (2009) 475-481.

[21] N. Verreyt, G.M. Nys, P. Santens, G. Vingerhoets, Cognitive differences between patients with left-sided and right-sided Parkinson's disease. A review, Neuropsychol. Rev. 21 (2011) 405-424.

[22] F.A. Barbieri, L. Simieli, D. Orcioli-Silva, A.M. Baptista, M.B. Pestana, V.S. Beretta, P.C.R. Santos, L.T.B. Gobbi, Obstacle avoidance increases asymmetry of crossing step in individuals with Parkinson's disease and neurologically healthy individuals, J. Mot. Behav. 49 (2017) 1-9.
[23] K. Martinu, A. Nagano-Saito, S. Fogel, O. Monchi, Asymmetrical effect of levodopa on the neural activity of motor regions in PD, PLoS One 9 (2014) e111600.

[24] V.S. Beretta, L.T.B. Gobbi, E. Lirani-Silva, L. Simieli, D. Orcioli-Silva, F.A. Barbieri, Challenging postural tasks increase asymmetry in patients with Parkinson's disease, PLoS One 10 (2015) e0137722.

[26] F.A. Barbieri, P.F. Polastri, A.M. Baptista, E. Lirani-Silva, L. Simieli, D. Orcioli-Silva, V.S. Beretta, L.T.B. Gobbi, Effects of disease severity and medication state on postural control asymmetry during challenging postural tasks in individuals with Parkinson's disease, Hum. Mov. Sci. 46 (2016) 96-103.

[27] A.J. Kolarik, A.C. Scarfe, B.C. Moore, S. Pardhan, An assessment of auditory-guided locomotion in an obstacle circumvention task, Exp. Brain Res. 234 (2016) 1725-1735.

[28] A.J. Hughes, S.E. Daniel, L. Kilford, A.J. Lees, Accuracy of clinical diagnosis of idiopathic Parkinson's disease: a clinico-pathological study of 100 cases, J. Neurol. Neurosurg. Psychiatry 55 (1992) 181-184.

[29] M.M. Hoehn, M.D. Yahr, Parkinsonism: onset, progression and mortality, Neurology 17 (1967) 427-442.

[30] M. Schenkman, C. Wei Zhu, T.M. Cutson, K. Whetten-Goldstein, Longitudinal evaluation of economic and physical impact of Parkinson's disease, Parkinsonism Relat. Disord. 8 (2001) 41-50.

[31] S. Fahn, R. Elton, UPDRS, Development Comitee Members of the, The unified parkinson's disease rating scale, in: S. Fahn, C.D. Marsden, D.B. Calne, M. Goldstein (Eds.), Recent Developments in Parkinson's Disease, McMellam Health Care Information, Florham Park, 1987, pp. 153-163.

[32] O.P. Almeida, Mini mental state examination and the diagnosis of dementia in Brazil, Arq. Neuropsiquiatr. 56 (1998) 605-612.

[33] S. Brucki, R. Nitrini, P. Caramelli, P.H. Bertolucci, I.H. Okamoto, Suggestions for utilization of the mini-mental state examination in Brazil, Arq. Neuropsiquiatr. 61 (2003) 777-781.

[34] D.A. Winter, Biomechanics and Motor Control of Human Movement, Wiley, Canada, 2005.

[35] L. Vallis, B. Mcfadyen, Locomotor adjustments for circumvention of an obstacle in the travel path, Exp. Brain Res. 152 (2003) 409-414.

[36] M. Gérin-Lajoie, C.L. Richards, B.J. McFadyen, The negotiation of stationary and moving obstructions during walking: anticipatory locomotor adaptations and preservation of personal space, Mot. Control 9 (2005) 242-269.

[37] J.M. Hausdorff, J.D. Schaafsma, Y. Balash, A.L. Bartels, T. Gurevich, N. Giladi, Impaired regulation of stride variability in Parkinson's disease subjects with freezing of gait, Exp. Brain Res. 149 (2003) 187-194.

[38] J.J. Silva, F.A. Barbieri, L.T.B. Gobbi, Adaptive locomotion for crossing a moving obstacle, Mot. Control 15 (2011) 419-433.

[39] R. Vitório, E. Lirani-Silva, F.A. Barbieri, V. Raile, F. Stella, L.T.B. Gobbi, Influence of visual feedback sampling on obstacle crossing behavior in people with Parkinson's disease, Gait Posture 38 (2013) 330-334.

[40] J.P. Azulay, S. Mesure, B. Amblard, O. Blin, I. Sangla, J. Pouget, Visual control of locomotion in Parkinson's disease, Brain 122 (1999) 111-120.

[41] Q.J. Almeida, J.S. Frank, E.A. Roy, M.E. Jenkins, S. Spaulding, A.E. Patla, M.S. Jog, An evaluation of sensorimotor integration during locomotion toward a target in Parkinson's disease, Neuroscience 134 (2005) 283-293.

[42] G. Dirnberger, M. Jahanshahi, Executive dysfunction in Parkinson's disease: a review, J. Neurol. 7 (2013) 193-224.

[43] J. Konczak, D.M. Corcos, F. Horak, H. Poizner, M. Shapiro, P. Tuite, M. Maschke, Proprioception and motor control in Parkinson's disease, J. Mot. Behav. 41 (2009) 543-552.

[44] K.A. Ehgoetz-Martens, Q.J. Almeida, Dissociating between sensory and perceptual deficits in PD: more than simply a motor deficit, Mov. Disord. 27 (2012) 387-392.

[45] A. Cronin-Golomb, Parkinson's disease as a disconnection syndrome, Neuropsychol. Rev. 20 (2010) 191-208.

[46] L. Hak, H. Houdijk, F. Steenbrink, A. Mert, P. van der Wurff, P.J. Beek, J.H. van Dieën, Speeding up or slowing down?: Gait adaptations to preserve gait stability in response to balance perturbations, Gait Posture 36 (2012) 260-264.

[47] A.L. Hof, M.G.J. Gazendam, W.E. Sinke, The condition for dynamic stability, J. Biomech. 38 (2005) 1-8.

[48] F.A. Barbieri, P.C.R. dos Santos, L. Simieli, D. Orcioli-Silva, J.H. van Dieën, L.T.B. Gobbi, Interactions of age and leg muscle fatigue on unobstructed walking and obstacle crossing, Gait Posture 39 (2014) 985-990.

[49] L. Simieli, L.T.B. Gobbi, D. Orcioli-Silva, V.S. Beretta, P.C.R. Santos, A.M. Baptista, F.A. Barbieri, The variability of the steps preceding obstacle avoidance (approach phase) is dependente on the height of the obstacle in people with Parkinson's Disease, PLoS One 12 (9) (2017) e0184134.

[50] J.M. Hausdorff, D.A. Rios, H.K. Edelberg, Gait variability and fall risk in community-living older adults: a 1-year prospective study, Arch. Phys. Med. Rehab. 82 (2001) 1050-1056.

[51] G.C. McIntosh, S.H. Brown, R.R. Rice, M.H. Thaut, Rhythmic auditory-motor facilitation of gait patterns in patients with Parkinson's disease, J. Neurol. Neurosurg. Psychiatry 62 (1997) 22-26.

[52] A. Nagy, G. Eördegh, Z. Paróczy, Z. Márkus, G. Benedek, Multisensory integration in the basal ganglia, Eur. J. Neurosci. 24 (2006) 917-924.

[53] S. Coren, Sensorimotor performance as a function of eye dominance and handedness, Percept. Mot. Skills 88 (1999) 424-426.

[54] J.R. Tresilian, The accuracy of interceptive action in time and space, Exerc. Sport Sci. Rev. 32 (2004) 167-173.

[55] A.C. Lee, J.P. Harris, E.A. Atkinson, M.S. Fowler, Disruption of estimation of bodyscaled aperture width in Hemiparkinson's disease, Neuropsychologia 39 (2001) 1097-1104.

[56] M.B. Knable, D.W. Jones, R. Coppola, T.M. Hyde, K.S. Lee, J. Gorey, 
D.R. Weinberger, Lateralized differences in iodine-123-IBZM uptake in the basal ganglia in asymmetric Parkinson's disease, J. Nucl. Med. 36 (1995) 1216-1225.

[57] K. Tatsch, J. Schwarz, P.D. Mozley, R. Linke, O. Pogarell, W.H. Oertel, H.F. Kung, Relationship between clinical features of Parkinson's disease and presynaptic dopamine transporter binding assessed with [123I] IPT and single-photon emission tomography, Eur. J. Nucl. Med. 24 (1997) 415-421.

[58] S.H. Johnson-Frey, The neural bases of complex tool use in humans, Trends Cogn. Sci. 8 (2004) 71-78.

[59] F. Binkofski, G. Buccino, K. Zilles, G.R. Fink, Supramodal representation of objects and actions in the human inferior temporal and ventral premotor cortex, Cortex 40 (2004) 159-161.

[60] S. Davidsdottir, R. Wagenaar, D. Young, A. Cronin-Golomb, Impact of optic flow perception and egocentric coordinates on veering in Parkinson's disease, Brain 131 (2008) 2882-2893.

[61] X. Ren, R. Salazar, S. Neargarder, S. Roy, T.D. Ellis, E. Saltzman, A. Cronin-Golomb, Veering in hemi-Parkinson's disease: primacy of visual over motor contributions, Vision Res. 115 (2015) 119-127.

[62] J.P. Harris, E.A. Atkinson, A.C. Lee, K. Nithi, M.S. Fowler, Hemispace differences in the visual perception of size in left hemiParkinson's disease, Neuropsychologia 41
(2003) 795-807.

[63] A.K. Wright, G.W. Arbuthnott, The influence of the subthalamic nucleus upon the damage to the dopamine system following lesions of globus pallidus in rats, Eur. J. Neurosci. 26 (2007) 642-648.

[64] A. Feigin, M. Fukuda, V. Dhawan, S. Przedborski, V. Jackson-Lewis, M.J. Mentis, D. Eidelberg, Metabolic correlates of levodopa response in Parkinson's disease, Neurology 57 (2001) 2083-2088.

[65] R.C. Duvoisin, L.I. Golbe, Toward a definition of Parkinson's disease, Neurolog 39 (1989) 746.

[66] A. Kumar, S. Mann, V. Sossi, T.J. Ruth, A.J. Stoessl, M. Schulzer, C.S. Lee, [11C] DTBZ-PET correlates of levodopa responses in asymmetric Parkinson's disease, Brain 126 (2003) 2648-2655.

[67] J.V. Jacobs, F.B. Horak, Abnormal proprioceptive-motor integration contributes to hypometric postural responses of subjects with Parkinson's disease, Neuroscience 141 (2006) 999-1009.

[68] K.A. Ehgoetz-Martens, C.G. Ellard, O.J. Almeida, Dopaminergic contributions to distance estimation in Parkinson's disease: a sensory-perceptual deficit? Neuropsychologia 51 (2013) 1426-1434. 